\title{
A closed-loop and ergonomic control for prosthetic wrist rotation
}

\author{
Mathilde Legrand ${ }^{1}$, Nathanaël Jarrassé ${ }^{1}$, Florian Richer ${ }^{1}$ and Guillaume Morel $^{1}$
}

\begin{abstract}
Beyond the ultimate goal of prosthetics, repairing all the capabilities of amputees, the development line of upperlimb prostheses control mainly relies on three aspects: the robustness, the intuitiveness and the reduction of mental fatigue. Many complex structures and algorithms are proposed but no one question a common open-loop nature, where the user is the one in charge of correcting errors. Yet, closing the control loop at the prosthetic level may help to improve the three main lines of research cited above. One major issue to build a closed-loop control is the definition of a reliable error signal; this paper proposes to use body compensations, naturally exhibited by prostheses users when the motion of their device is inaccurate, as such. The described control scheme measures these compensatory movements and makes the prosthesis move in order to bring back the user into an ergonomic posture. The function of the prosthesis is no longer to perform a given motion but rather to correct the posture of its user while $s /$ he focus on performing an endpoint task. This concept was validated and compared to a standard open-loop scheme, for the control of a prosthetic wrist, with five healthy subjects completing a dedicated task with a customized transradial prosthesis. Results show that the presented closed-loop control allows for more intuitiveness and less mental burden without enhancing body compensation.
\end{abstract}

\section{INTRODUCTION}

Upper limb prostheses aim at replacing the amputated limb of their users, to give them back some autonomy and capabilities. This substitution calls on developments in two main areas: mechatronics and control. Mechatronics now provides devices with multiple degrees of freedom (DOF), such as polydigital hands [1], [2], 2-DOF wrist [3] or whole multiple DOF arm[4] but the control of these devices still remains a challenge.

Two main control approaches exist: myoelectric and movement-based; each one tries to bring a solution to the identified issues of prosthetic control, such as intuitiveness, reduced fatigue and mental burden, or robustness [5]-[8]. The most common one, used in daily life, is conventional myoelectric control, that takes the electrical signals of some flexor and extensor muscles of the residual limb to control the actuators of an externally-powered prosthetic joint [9]. When there are several DOF to control, the user cannot make a simultaneous motion but has to switch from one joint to another, either with a co-contraction or by modulating finely the muscle contraction amplitude with proportional control approaches [10]. This sequential control is cumbersome and

\footnotetext{
*This work was supported by ANR Byceps, ANR-18-CE19-0004

${ }^{1}$ Mathilde Legrand, Nathanaël Jarrassé, Florian Richer and Guillaume Morel are with Institute of Intelligent Systems and Robotics, Sorbonne University, Paris, France \{mathilde.legrand, nathanael.jarrasse, florian.richer, guillaume.morel\}@sorbonne-universite.fr
}

leads to muscular fatigue [5]. To overtake these limitations, pattern recognition relies on muscular synergies to simplify the access to several joint motions and to reduce mental burden [8], [11], [12], but without solving the issues of robustness and easy access to good electromyogram measurements. Movement-based control techniques choose to make use of joints motions instead of muscles signals, claiming for more intuitiveness and less complexity in signal processing. Some of them artificially link the motion of one functional joint to a prosthetic joint (joint-to-joint correspondence) [13], [14], others use functional joints as joystick to control the end-effector trajectory (for shoulder amputees only) [15] and others employ models of joint synergies [16]-[18].

Each of these techniques has pros and cons but one major issue remains common to all: they are open-loop control schemes. Whether the prosthesis motion corresponds to the user's intention is never considered by the system itself. Each technique leaves this correction step to the user, which increases his/her mental burden and leads to a high reaction time. To improve this aspect, two approaches can be developped: either optimizing the control algorithms in order to eliminate every possible errors, or finding ways to close the control loop. The first approach seems unrealistic: first, because achieving an effective intention decoding is a very complex challenge (particularly because of human variability); second because even the slightest error can discourage an everyday prosthesis user. Considering pattern recognition for instance, an algorithm that would achieve 99\% of correct outputs would probably not be good enough: the $1 \%$ of error could put the user in uncomfortable or dangerous situations and thus still ask for a correction from the user him/herself. Alternatively, the closed-loop approaches may have not been developped yet because of the hurdle to define an error signal as the goal of the task is rarely known to the robotic limb. Indeed, how to perfectly determine the task to perform or the object to grasp in an unmonitored environment, where the only reliable source of information is the amputee him/herself and when the humanrobot communication channel relies on very limited signals (muscular contractions or joint motions)? An attempt to identify the intended motion is gaze-tracking-based solutions [19]. Still, they are not very accurate - making the control not very robust - because of the resolution of the cameras used and the intrinsic human eye limitation [20]. They also force the user to lock his/her gaze on the target and to constantly wear eye-tracker glasses.

When the movement of a prosthesis does not perfectly correspond to its user's intention, a common behavior stands out: the user tends to exhibit compensatory motions, in 


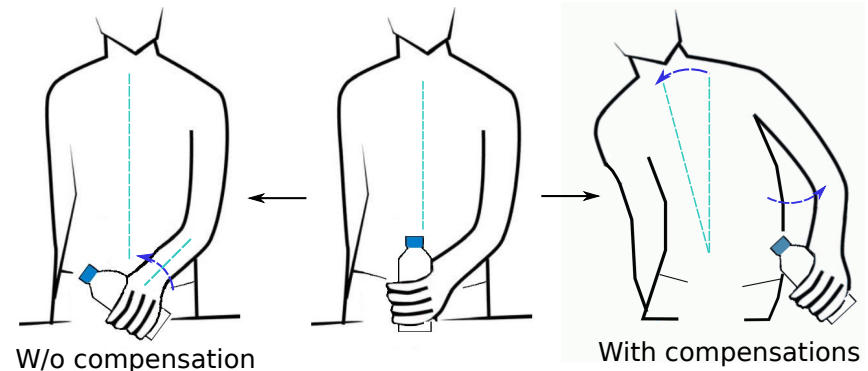

Fig. 1: Illustration of typical body compensations (use of trunk and upper-arm) exhibited to counteract an impaired or motionless wrist when rotating an object.

order to compensate the unadapted position/orientation of the end-effector [21], [22] (see Figure 1). This often allows for faster, more accurate and less cumbersome correction than re-sending input signals (e.g. muscle contractions). We propose here to use these body compensations as an error signal to close the control loop. Oyama et al., in [23], had build a control law for wrist prono-supination that aimed at minimizing body compensations. Yet, there is no error or closed-loop considerations, since the wrist rotation is merely proportional to a weighted combination of shoulder angles. We rather focus on these two aspects and consider that the function of the prosthesis is not to perform a given motion but to correct the posture of its user, while letting him/her executing an endpoint task. The control of the end-effector grasping function is then counted as a separate case.

In this paper, we present this concept we called ergonomic control, and apply it to the pronosupination of a prosthetic wrist. We setup an experiment in which we compare a standard open-loop with our closed-loop control. Considering that body compensations are physiologically armful and may lead to musculo-skeletal disorders [24], [25], this study does not only verify that the proposed concept is functional but also that it does not enhance compensatory motions, as suggested by recent upper limb motions assessments [26][28].

\section{METHODS}

\section{A. Closed-loop ergonomic control for wrist pronosupination}

Conventional myoelectric control is kept for the grasping function as it seems good enough when used to control only the hand; the difficulty arises when additional DOF are added. We are thus interested in testing first our control approach on the wrist joint.

Our hypothesis is that, if a prosthesis does not move or not as expected, a given task is still achieved by the subject but with body compensations. The approach described here, ergonomic control (EC), detects and employs these compensatory motions to control the robotic joint, whose motion will then make the user go back to a comfortable ergonomic position while $\mathrm{s} / \mathrm{he}$ is performing the task.

When wrist pronosupination is absent or not correct, it can be supplanted by trunk and arm compensatory motions

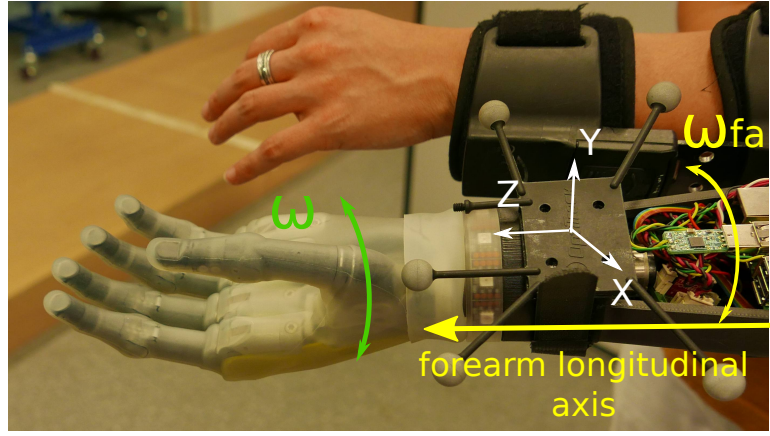

Fig. 2: Diagram showing the definition of the reference frame used to compute XYZ Euler angles and obtained $\epsilon$, and the definition of $\omega$.

[21], [22], [26]. However, (i) these compensations may differ depending on the subject's posture and (ii) all trunk and arm motions are not compensations. We could artificially map trunk and arm movements to the prosthesis' motions but this would create rigid coordinations between functional and robotic joints and limit the overall mobility of the subject. We worked instead at a more distal level. To complete the uncorrect or replace the missing wrist pronosupination, trunk and arm compensatory motions aim at changing the hand orientation, rotating the forearm around its longitudinal axis (elbow-hand axis). We directly measure this rotation since it simplifies the data to record and thus the type and the number of sensors to use.

When the forearm orientation is modified, it means that trunk and/or arm are moving to compensate; prosthetic wrist has to move to make the subject going back to a more ergonomic posture. The error signal is then set as

$$
\epsilon(t)=\omega_{f a}(t)-\omega_{0}
$$

with $\omega_{f a}$ the forearm rotation angle around its longitudinal axis, i.e. the Z-Euler angle of XYZ sequence (see Figure 2), and $\omega_{0}$ a reference forearm orientation, which defines the ergonomic posture to go back to. The reference definition is set here as the initial forearm orientation but can change if necessary, depending on the task. This generated error vector is simpler to measure than any spatial error related to an action in space.

$\epsilon$ can be used as the input of a simple control law, which pilots the prosthetic wrist angular velocity, $\dot{\omega}$, as defined in the following equation:

$$
\dot{\omega}(t)=\left\{\begin{array}{l}
0 \text { if }|\epsilon(t)|<\epsilon_{0} \\
\lambda\left(\epsilon(t)-\delta \epsilon_{0}\right) \text { otherwise, }
\end{array}\right.
$$

where $\epsilon_{0}$ is a deadzone threshold (here set to $5 \mathrm{deg}$ ), $\delta=$ $\operatorname{sign}\left(\epsilon(t)-\epsilon_{0}\right)$, and $\lambda$ is a scalar gain (set to 2 ) that tunes the rate of correction. The frequency of the control loop is $100 \mathrm{~Hz}$. The values of $\epsilon_{0}$ and $\lambda$ were chosen experimentally to ensure stability. 


\section{B. Experimental set-up}

Five healthy subjects (two females and three males, 22 to 25 years old, one left-handed and four right-handed) performed the wire loop task of the Powered Arm Prosthetic Race of the Cybathlon(c)[29]. The goal of the task is to bring the handle through the wire loop in a minimum time and without touching the wire. The shape of the wire requires the participants to continuously adjust their hand orientation with pronosupination. Attached to their left arm, the subjects wore a prosthetic prototype composed of a Touch Bionics hand (I-Limb ${ }^{\mathrm{TM}}$ Ultra) and a motorized wrist rotator. The hand was pre-positioned holding the handle and kept still during the experiment (see Figure 3); the wrist angular velocity was controlled by a DC motor driver (Ion motor control, Ltd), itself controlled by a Raspberry Pi 3(c). We used an Optitrack (NaturalPoints Inc.) motion capture system both for EC and for post-experiment analyses. Seven rigid bodies (or clusters), made of Optitrack markers, were tracked: two on the hips, one on the torso, two on the shoulders, one on the device's forearm and one on the handle. Two Inertial Measurement Units were also placed on the trunk and the upper arm respectively for post-experiment analyses (see Figure 4). Optitrack was convenient in lab environment and for post-experiment analyses but the data used for EC could have easily been replaced by gyroscope data from an Inertial Measurement Unit.

Two control schemes of the device were compared:

1) an open-loop control (OL). A pair of push-buttons mounted on a handle was grasped by the free hand of the subjects (see Figure 4(a)). One push-button controlled the forward wrist rotation, the other the backward rotation. The joint angular velocity was fixed at $50 \mathrm{deg} / \mathrm{s}$. When no push-button was pressed, the device stayed still. This command was used as a reference as it is a discrete open-loop control and thus mimicks most of the myoelectric controllers currently available for lower arm amputees (on/off, proportional switch or even pattern recognition) [30], while avoiding all the practical problems of EMG measurements [5], [31].

2) ergonomic control (EC). The forearm rotation, $\omega_{f a}$, was computed in the initial forearm frame, from quaternions of the Optitrack cluster placed on the device's forearm and sent in real time to the prosthesis. As subjects naturally tended to stay parallel to the wire during the whole experiment, the reference posture, $\omega_{0}$, was supposed to be fixed and was set as the initial position of the forearm. For more complex or changing tasks, the reference shall be updated, either in a very simple way, by the user himself with an external signal, or with a dedicated algorithm.

Before each recording session, subjects were allowed to train a few times on the wire loop task. They trained between two and five times. A recording session consisted in five trials and there were three recording sessions: one "natural" (N) with the own left forearm of the subjects, which provides a baseline from natural motions, one with

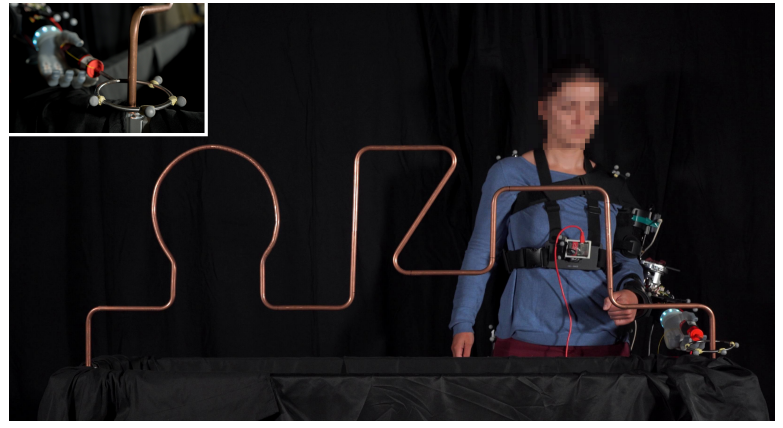

Fig. 3: Cybathlon wire-loop task with the adapted prosthetic device. The upper left insert shows the handle hold by the prosthetic hand.

OL, one with EC. Subjects always began with $\mathrm{N}$ while EC/OL order was randomized, to avoid measuring any effect of task learning.

Post-experiment analyses were conducted with Matlab, Mathworks Inc. This experiment was carried out in accordance with the recommendations of Universite Paris Descartes ethic committee CERES, which approved the protocol. All participants gave their written informed consent in accordance with the Declaration of Helsinki.

\section{RESULTS}

EC was assessed on several criteria and compared to the standard OL control. The metrics characterize the good realization of the task but also the smoothness of the movement and the amount of generated body compensations.

\section{A. Task realization}

The task performance was described with the time of the task and the number of touches (see Figure 5). Figure 5(a) shows the time for each trial of the two modes, with the mean difference between the two modes and the $95 \%$ confidence intervals (CI). This representation was selected because it allows for a more transparent statistical analysis than the pvalue [32], especially for small populations. If the $95 \%$ CI of the mean difference does not cross the zero dotted line, the two modes are statistically significantly different. The green lines are the minimum and maximum times obtained with $\mathrm{N}$, during natural achievement of the task by each subject. It can be noticed that the natural times of the task are shorter for $\mathrm{S} 1$ because this subject was left-handed, but handedness did not affect any other results. We see here that for three out of five participants (S1, S2 and S4), the time is statistically significantly shorter with EC than with OL. For S5, the time with EC is longer but without a real significant difference. S3 stands out from the others as EC times are significantly longer than OL ones. On Figure 5(b), we observe that the number of touches does not really distinguish OL and EC, except for $S 1$. The lattest touched the wire in four out of five trials with OL and never with EC, but this is partly due to the fact that this subject wanted to be fast before being precise. Overall, it can also be noticed that EC generates touches in 

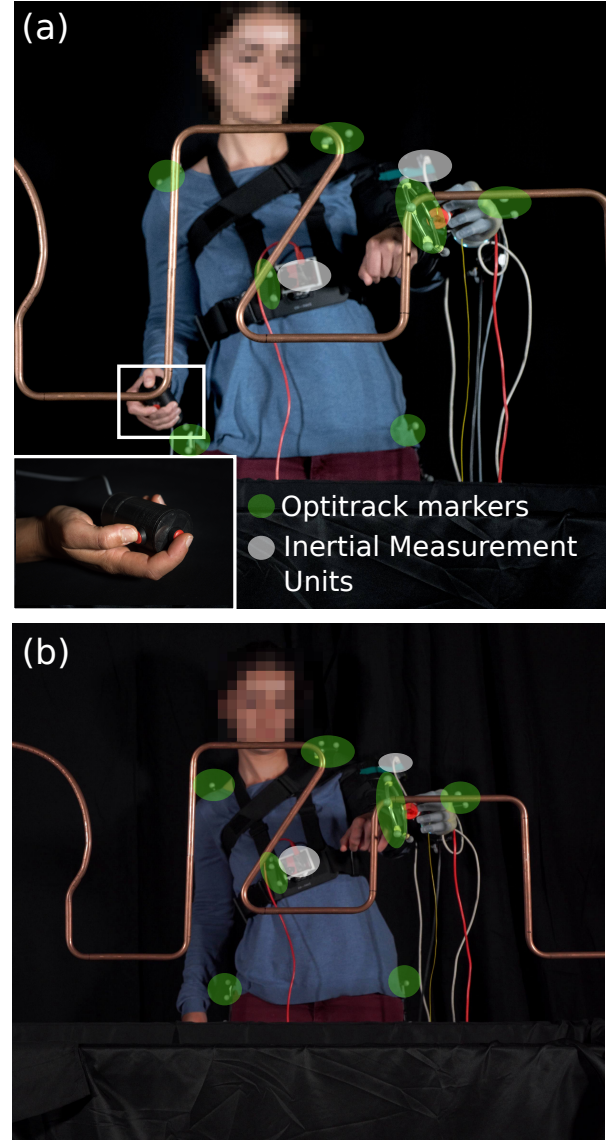

Fig. 4: Experimental set-up. (a) OL control set-up. (b) EC set-up. 1: Optitrack markers; 2: Inertial Measurement Units.

only one trial out of the 25 (total of all trials for all subjects) whereas OL does in 7 trials.

\section{B. Smoothness of wrist motions}

While healthy motions are naturally smooth [33], prosthetic motions are often jerky, due to the discrete nature of the command [34]. Since EC allows for a continuous control of the movements, we infer that the smoothness of prosthetic motions with EC is improved, which would be a significant benefit. Figure 6 shows the wrist pronosupination angle and the wrist angular velocity of the prosthesis for one typical subject, for OL and EC respectively. We see on Figure 6(a) that the OL wrist angular velocity must be a series of pulses to obtain a motion as continuous as possible. Yet, it is clear that the wrist angular trajectory with OL is jerky (it is a step function) while the one with EC is smooth and continuous (see Figure 6(b)). This difference in smoothness can be illustrated by the high number of times the push-buttons were pressed compared to the low number of deadzone crossings with EC. These measures can also represent the involvement required from the subject, as pressing the push-buttons or exhibiting compensations to move the wrist increase the mental charge. Figure 7(a) shows these two metrics, with their mean and standard deviation, for all trials of the five participants. It highlights the fact that the prosthesis user is (a)

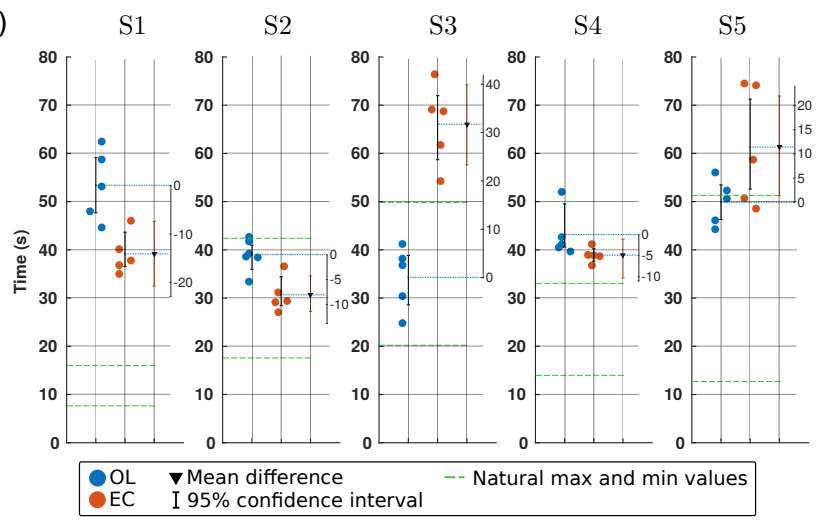

(b)

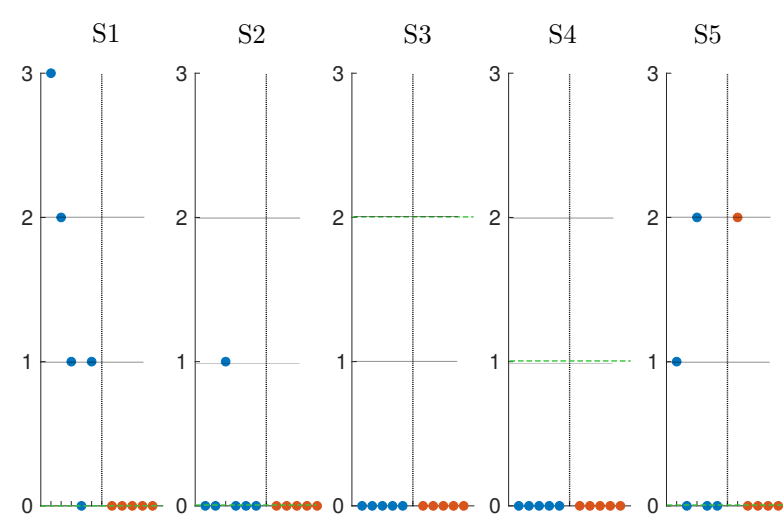

Fig. 5: Task realization performance metrics. (a) Time of the task: all trials of each of the five subjects, with the $95 \%$ confidence interval and the mean difference between the two control modes. (b) Number of handle touching the wire for each trial. The green lines are (a) the maximum and minimum values of the time and (b) the maximum number of touches when the subject performed the task with his/her own hand. OL is open-loop and EC ergonomic control.

much more involved when using OL than when using EC: (i) $s /$ he has to focus on individual wrist motions while EC allows to focus only on the end-effector motion and (ii) s/he is her/himself the corrector that closes the control loop.

To quantitatively evaluate smoothness, we used the spectral arc length of the wrist angular velocity [35] (Figure 7(b), the more negative, the less smooth). The same statistical representation as above, with $95 \%$ confidence intervals and difference mean, was employed. There is no doubt that for the five participants, EC wrist motions are significantly smoother than OL wrist motions.

Yet, one can criticize the fact that the low smoothness of OL is intrinsic to its binary nature. As often done in on/off prosthetic control, finer velocity profiles, e.g. trapezoidal, could have been implemented. However, this would not have created a fully continuous control, as enabled by EC.

\section{Joint motions}

Another important point in upper limb motions assessment is to which extent the subject has to modify his/her joint movements and coordinations. It is particularly relevant in 
(a)

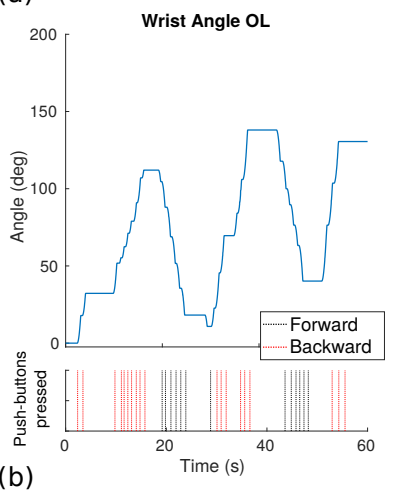

(b)

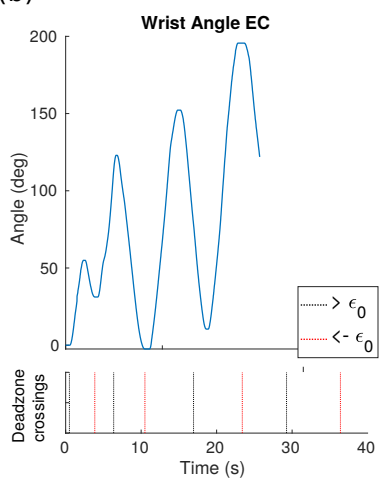

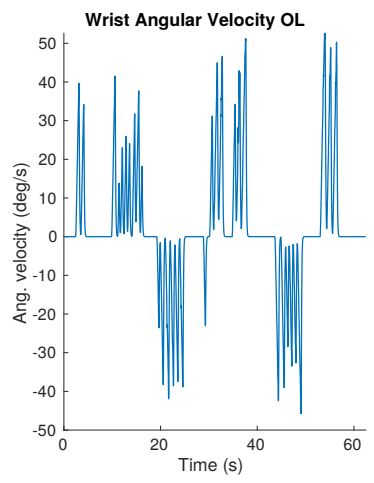

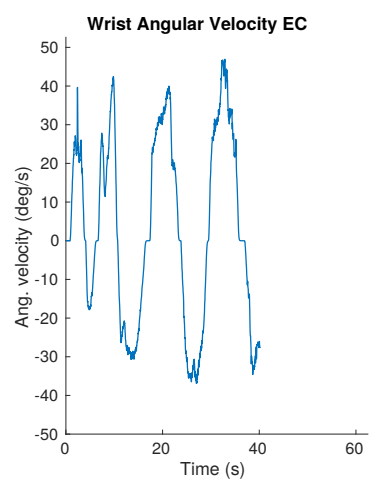

Fig. 6: Prosthetic wrist pronosupination angle and angular velocity for one trial of one typical subject with (a) open-loop control and (b) ergonomic control. The dashed lines indicate when the push-buttons are pressed (OL) or when the subject's forearm angle went out of the deadzone (EC).

our case, where one may think that, due to its intrinsic characteristics, EC could potentially increase some trunk and arm compensatory motions. We focus on the study of the arm aperture angle, $\alpha$, and the lateral bending of the trunk, $\psi$ (see definitions on Figure 8(a)), to verify that the action of the prosthesis is fast enough to prevent any important and undesirable compensations.

Figure $8(\mathrm{~b})$ is the mean and standard deviation of the angular trajectories, normalized in time, for each participant. Green dashed lines represent the maximum and minimum values of each subject's natural angular trajectories. We first note that the timing of the angular variations are similar between the two modes of control. $\alpha$ (up panel) shows variations of amplitude between OL and EC but, except for S4, both always stay into the subject's natural baseline. For S4, even if $\alpha$ with EC shows higher values than with OL and exceeds the individual's natural values, the maximum is still lower than some natural maximum of other subjects. Contrary to $\alpha, \psi$ is a bit heightened with EC, especially for left-side bending (negative values). This observation points out the issue of the reference definition, which can affect the exhibited compensatory motions. Indeed, prosthetic wrist pronation is induced either by arm internal aperture (internal $\alpha$, see Figure $8(\mathrm{a})$ ) or by trunk left bending (negative $\psi$ ). Because we chose, for this experiment, the reference as
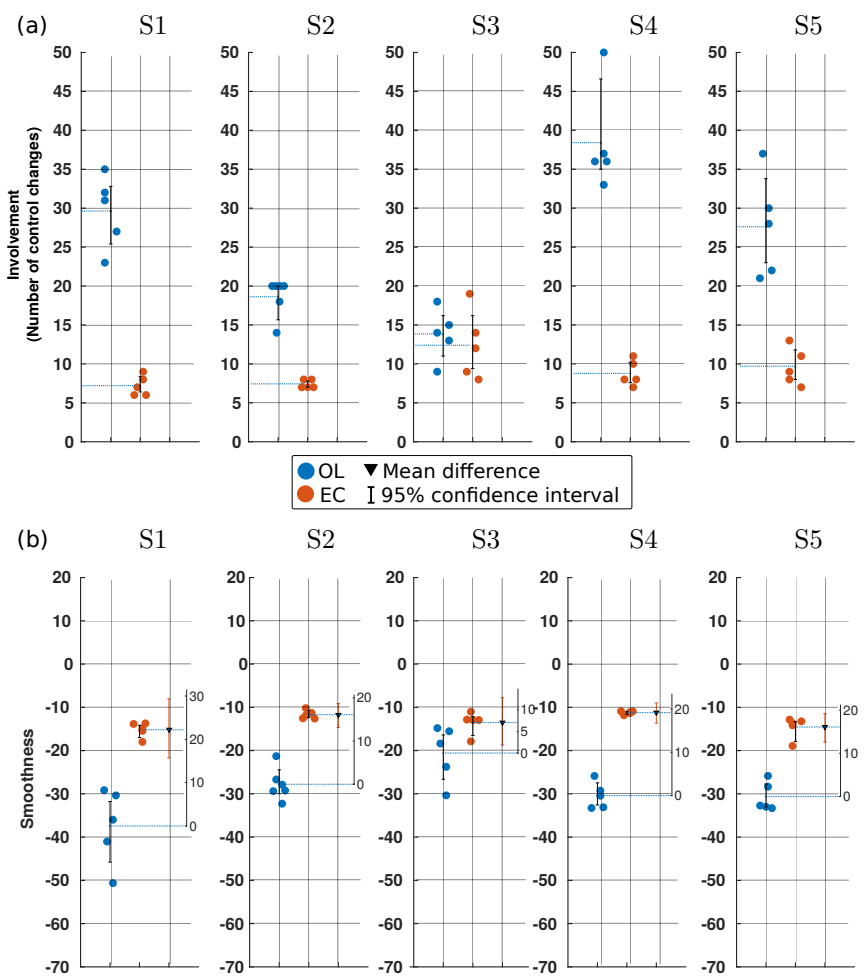

Fig. 7: Wrist motion metrics. (a) Involvement of the subjects measured by the number of times the push-buttons are pressed (OL) and the number of deadzone crossings (EC). (b) Motion smoothness measured by the spectral arc length of wrist angular velocity: $95 \%$ of confidence interval and mean difference between the two control modes.

the initial position of the subject, with the arm along the body, the amplitude of internal $\alpha$ was limited. The latter was so difficult to perform that it was nearly not exhibited and, to induce wrist pronation, the subject used only left lateral bending. This phenomenon can be prevented by optimizing the choice of the reference position. If subjects were given the instruction to begin with a small external $\alpha$, the amplitude of internal angle would have been increased, avoiding a too important use of the trunk to obtain pronation. We are currently working to find a reference posture that does not promote pronation or supination but allows an easy realization of both without enhancing trunk or arm compensations.

\section{CONCLUSIONS}

Ergonomic control was used with a 1-DOF prosthetic wrist, by five subjects performing a dedicated task, the wire loop of Powered Arm Prosthetic Race of the Cybathlon (c). EC was compared to OL, a discrete open-loop control that mimicks existing on/off prosthetic control, like myoelectric. EC shows better results than OL for the time of the task, the number of touches and also for mental burden, which is a great advantage over existing controls of prostheses. 
(a)

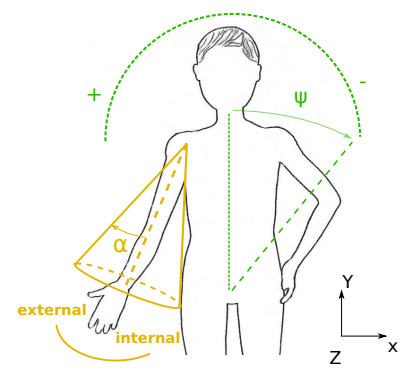

(b)

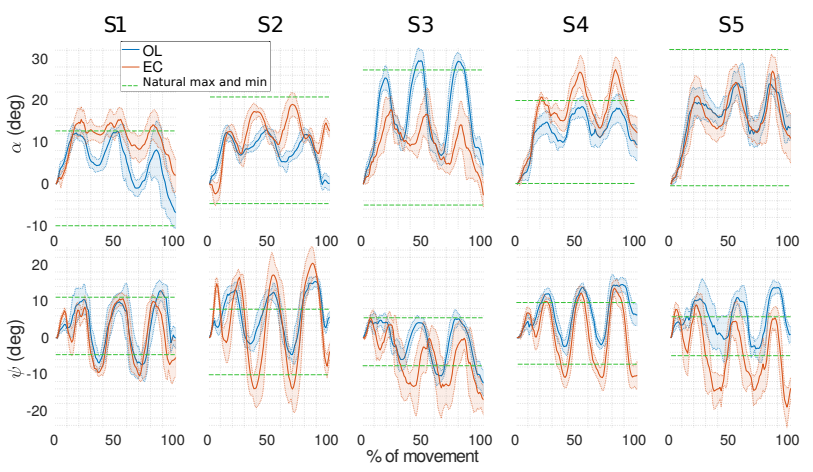

Fig. 8: Arm and trunk motions. (a) Definition of arm aperture and trunk lateral bending angles. (b) Mean and standard deviation of $\alpha$ (up panel) and $\phi$ (down panel), normalized in time, for all subjects. Green dashed lines are the maximum and minimum values of the subject's natural

joint trajectories. OL is open-loop and EC ergonomic control.

Moreover, closing the control loop on body compensations naturally reduces them.

The wire loop experiment thus illustrates the interest for closed-loop ergonomic control for an artificial pronosupination joint. It exploits natural strategies of compensatory movements to close the control loop, without any necessity to know the environment or the task. The two main advantages of the proposed control are its intuitiveness and its simplicity. Indeed, it was mastered by all the participants without considerable training session and the control law has only two parameters to tune (the threshold, $\epsilon_{0}$, and the gain, $\lambda)$. This ensures easy generalization to all users as well as robustness. The prosthesis user only has to focus on the endoint task while the prosthesis corrects his/her posture. EC is thus much less cumbersome than most of the existing open-loop control schemes with which the user has to focus on the motions of individual joints.

The validation of ergonomic control was performed here on healthy subjects. Upcoming developments will thus include in-depth study with amputated participants. EC was also tested on only one task and for one DOF. Future works will aim at using Inertial Measurement Units instead of motion capture system for an easy transfer to daily life (raw gyroscope values can be sufficient, avoiding drift issues), studying EC use with a broader set of tasks and adapting it to more DOF (e.g. adding the elbow joint). This should allow for a simultaneous control of several prosthetic joints without more mental burden for the user.

\section{ACKNOWLEDGMENT}

We thank all the participants of the experiment.

\section{REFERENCES}

[1] F. Cordella, A. L. Ciancio, R. Sacchetti, A. Davalli, A. G. Cutti, E. Guglielmelli, and L. Zollo, "Literature review on needs of upper limb prosthesis users," Front. Neurosci., vol. 10, no. 209, 2016.

[2] C. Piazza, M. Catalano, S. Godfrey, M. Rossi, G. Grioli, M. Bianchi, K. Zhao, and A. Bicchi, "The softhand pro-h," IEEE Robot. Autom. Mag., 2017.

[3] P. J. Kyberd, E. D. Lemaire, E. Scheme, C. MacPhail, L. Goudreau, G. Bush, and M. Brookeshaw, "Two-degree-of-freedom powered prosthetic wrist," J. Rehabil. Res. Dev., vol. 48, pp. 609-618, 2011.

[4] L. Resnik, S. L. Klinger, and K. Etter, "The deka arm: Its features, functionality, and evolution during the veterans affairs study to optimize the deka arm," Prosthet.Orthot. Int., vol. 38, pp. 492-504, 2014.

[5] C. Castellini, P. Artemiadis, M. Wininger, A. Ajoudani, M. Alimusaj, A. Bicchi, B. Caputo, W. Craelius, S. Dosen, K. Englehart, D. Farina, A. Gijsberts, S. B. Godfrey, L. Hargrove, M. Ison, T. Kuiken, M. Marković, P. M. Pilarski, R. Rupp, and E. Scheme, "Proceedings of the first workshop on peripheral machine interfaces: Going beyond traditional surface electromyography," Front. Neurorobot., vol. 8, pp. $1-17,2014$.

[6] H. L. Benz, J. Yao, L. Rose, O. Olgac, K. Kreutz, A. Saha, and E. F. Civillico, "Upper extremity prosthesis user perspectives on unmet needs and innovative technology," in Conf. Proc. IEEE Eng. Med. Biol. Soc., vol. , 2016, pp. 287-290.

[7] P. Beckerle, G. Salvietti, R. Unal, D. Prattichizzo, S. Rossi, C. Castellini, S. Hirche, S. Endo, H. Amor, M. Ciocarlie, F. Mastrogiovanni, B. Argall, and M. Bianchi, "A human-robot interaction perspective on assistive and rehabilitation robotics," Front. Neurorobot. vol. 11, 2017.

[8] I. Vujaklija, D. Farina, and O. Aszmann, "New developments in prosthetic arm systems," Orthop. Res. Rev., vol. 8, pp. 31-39, 2016.

[9] B. Popov, "The bio-electrically controller prosthesis," Journal of Bone and Joint Surgery, vol. 47, pp. 421-424, 1965.

[10] A. D. Roche, H. Rehbaum, D. Farina, and O. C. Aszmann, "Prosthetic myoelectric control strategies: A clinical perspective," Curr. Surg. Rep., vol. 2, 2014.

[11] P. Herberts, C. Almstrm, R. Kadefors, and P. D. Lawrence, "Hand prosthesis control via myoelectric patterns," Acta Orthop. Scand., pp. 389-409, 1973

[12] M. A. Oskoei and H. Huosheng, "Myoelectric control systems-a survey," Biomed. Signal Process. Control, vol. 2, 2007.

[13] D. A. Bennett and M. Goldfarb, "Imu-based wrist rotation control of a transradial myoelectric prosthesis," IEEE Trans. Neural Syst. Rehabil. Eng., vol. 26, no. 2, pp. 419-427, 2017.

[14] L. Resnik, S. L. Klinger, K. Etter, and C. Fantini, "Controlling a multidegree of freedom upper limb prosthesis using foot controls: User experience," Disabil. Rehabil. Assist. Technol., vol. 9, no. 4, pp. 318329, 2014.

[15] S. L. Phillips, L. Resnik, C. Fantini, and G. Latlief, "Endpoint control for a powered shoulder prosthesis," J. Prosthet. Orthot., vol. 25, no. 4, pp. 193-200, 2013

[16] M. Merad, E. de Montalivet, A. Roby-Brami, and N. Jarrassé, "Intuitive prosthetic control using upper limb inter-joint coordinations and imu-based shoulder angles measurement: A pilot study," in Proc. IEEE/RSJ Int. Conf. Intelligent Robots and Systems, 2016.

[17] S. Iftime, L. Egsgaard, and M. Popovic, "Automatic determination of synergies by radial basis function artificial neural networks for the control of a neural prosthesis," IEEE Trans. Neural Syst. Rehabil. Eng., vol. 13 , no. 4, pp. 482-489, 2005.

[18] M. Farokhzadi, A. Maleki, A. Fallah, and S. Rashidi, "Online estimation of elbow joint angle using upper arm acceleration: A movement partitioning approach," J. Biomed. Phys. Eng., vol. 7, no. 3, pp. 305314, 2016.

[19] M. R. Maymo, A. Shafti, and A. A. Faisal, "Fastorient: Lightweight computer vision for wrist control in assistive robotic grasping," in Proc. IEEE RAS EMBS Int. Conf. Biomed. Robot. Biomechatron. 2018. 
[20] A. Kar and P. Corcoran, "Towards the development of a standardized performance evaluation framework for eye gaze estimation systems in consumer platforms," in Proceedings of the IEEE International Conference on Systems, Man and Cybernetics.

[21] S. L. Carey, M. Jason Highsmith, M. E. Maitland, and R. V. Dubey, "Compensatory movements of transradial prosthesis users during common tasks," Clin. Biomech., vol. 23, pp. 1128-1135, 2008.

[22] A. J. Metzger, A. W. Dromerick, R. J. Holley, and P. S. Lum, "Characterization of compensatory trunk movements during prosthetic upper limb reaching tasks," Arch. Phys. Med. Rehabil., vol. 93, pp. 2029-2034, 2012.

[23] S. Oyama, S.and Shimoda, F. S. K. Alnajjar, K. Iwatsuki, M. Hoshiyama, H. Tanaka, and H. Hirata, "Biomechanical reconstruction using the tacit learning system: Intuitive control of prosthetic hand rotation," Front. Neurorobot., 2016.

[24] C. Gambrell, "Overuse syndrome and the unilateral upper limb amputee- consequences and prevention," J. Prosthet. Orthot., pp. 126132, 2008.

[25] K. Ostlie, R. J. Franklin, O. H. Skjeldal, A. Skrondal, and P. Magnus, "Musculoskeletal pain and overuse syndromes in adult acquired major upper-limb amputees," Arch. Phys. Med. Rehabil., vol. 92, pp. 19671973, 2011.

[26] K. Kontson, I. Marcus, B. Myklebust, and E. Civillico, "An integrated movement analysis framework to study upper limb function: A pilot study," IEEE Trans. Neural Syst. Rehabil. Eng., vol. 25, no. 10, pp. 1874-1883, 2017.

[27] M. Deijs, R. Bongers, N. Ringeling-Van Leusen, and C. Van Der Sluis, "Flexible and static wrist units in upper limb prosthesis users: Functionality scores, user satisfaction and compensatory movements," $J$. Neuroeng. Rehabil., vol. 13, pp. 1-13, 2016.

[28] A. Hussaini and P. Kyberd, "Refined clothespin relocation test and assessment of motion," Prosthet. Orthot. Int., pp. 294-302, 2017.

[29] Race Task Description Cybathlon 2020, ETH Zürich, 2020.

[30] D. Farina, N. Jiang, H. Rehbaum, A. Holobar, B. Graimann, H. Dietl, and O. Aszmann, "The extraction of neural information from the surface emg for the control of upper-limb prostheses: Emerging avenues and challenges," IEEE Transactions on Neural Systems and Rehabilitation Engineering, vol. 22, no. 4, pp. 797-809, 2014.

[31] J. Lobo-Prat, P. N. Kooren, A. H. Stienen, J. L. Herder, B. F Koopman, and P. H. Veltink, "Non-invasive control interfaces for intention detection in active movement-assistive devices." J. Neuroeng. Rehabil., vol. 11, 2014.

[32] J. Ho, T. Tumkaya, S. Aryal, H. Choi, and A. Claridge-Chang, "Moving beyond p values: data analysis with estimation graphics," Nature Methods, pp. 565-566, 2019.

[33] T. J. Sejnowski, "Making smooth moves," Nature, vol. 394, pp. 725726, 1998.

[34] J. Cowley, L. Resnik, J. Wilken, L. S. Walters, and D. Gates, "Movement quality of conventional prostheses and the deka arm during everyday tasks," Prosthetics and Orthotics International, 2016.

[35] S. Balasubramanian, A. Melendez-Calderon, and E. Burdet, "A robust and sensitive metric for quantifying movement smoothness," IEEE Trans. Biomed. Eng., vol. 59, no. 8, pp. 2126-2136, 2012. 